\title{
Separation of linear, mono-methyl and di-methyl alkanes in the 5-7 carbon atom range by exploiting configurational entropy effects during sorption on silicalite-1
}

\author{
S. Calero, B. Smit and R. Krishna*
}

Department of Chemical Engineering, University of Amsterdam, Nieuwe Achtergracht 166, 1018 WV Amsterdam, The Netherlands.E-mail:krishna@its.chem.uva.nl

\author{
Received 5th April 2001, Accepted 2nd August 2001 \\ First published as an Advance Article on the web 12th September 2001
}

\begin{abstract}
We have developed a novel concept for separating alkane mixtures, in the 5-7 carbon atom range, into three separate fractions consisting of linear, mono-methyl and di-methyl alkanes by adsorption on silicalite. We make use of the fact that with increased degree of branching, the "packing efficiency" within the silicalite matrix is significantly lower. This configurational entropy effect comes into play when the loading exceeds 4 molecules per unit cell, when all the intersection sites are occupied, and results in the following hierarchy of sorption strengths: linear alkanes $\gg$ mono-methyl alkanes $\gg$ di-methyl alkanes. To demonstrate the feasibility of the entropy-based separation concept we carried out configurational-bias monte carlo simulations for a variety of binary, ternary and quaternary mixtures of alkanes, n-pentane $\left(n-C_{5}\right), 2$-methylbutane $(2 \mathrm{MB})$, n-hexane (n-C $)_{6}$, 2-methylpentane (2MP), 3-methylpentane (3MP), 2,2-dimethylbutane (22DMB), n-heptane (n$\mathrm{C}_{7}$ ), 2-methylhexane (2MH), 2,2-dimethylpentane (22DMP). The entropy-driven separation concept has been verified, in part, by comparison with the experimental sorption data of Santilli (J. Catal., 1986, 99, 335) for the mixture $n-\mathrm{C}_{6}-3 \mathrm{MP}-22 \mathrm{DMB}$.
\end{abstract}

\section{Introduction}

In the petroleum industry catalytic isomerization is used to convert linear alkanes into their branched isomers. Isomerization processes generate a mixture of isomers that usually require separation and recycling of the non-isomerized components. For example, the effluent of a paraffin isomerization reactor may contain normal alkanes, mono-methyl alkanes and di-methyl alkanes. Traditionally, only the normal alkanes would be separated from the mixture by adsorption and recycled to the isomerization reactor, and any mono-methyl alkanes would be collected with the di-methyl alkanes as product. ${ }^{1}$ However, it is the di-methyl alkanes that are the most desired and have the highest octane number components. Consider for example the isomers of hexane; $n$ hexane $\left(\mathrm{n}-\mathrm{C}_{6}\right)$ has a RON (Research Octane Number) $=30$ whereas the corresponding $\mathrm{RON}$ values for its isomers are: 2-methylpentane $(2 \mathrm{MP})=74.5 ; 3$-methylpentane $(3 \mathrm{MP})=$ $75.5 ; 2,2$-dimethylbutane $(22 \mathrm{DMB})=94 ; 2,3$-dimethylbutane $(23 \mathrm{DMB})=105$. Therefore, a more efficient approach would be to adsorptively separate only the di-methyl alkanes as product and recycle the normal and the mono-methyl alkanes to the isomerization reactor. ${ }^{1}$

The separation of alkane mixtures is usually achieved by selective adsorption within zeolitic materials. ${ }^{1-3}$ The choice of the zeolite is largely governed by the sorption selectivity $S$; for the separation of components 1 and $2, S$ is defined as

$$
S=\frac{\Theta_{2} / \Theta_{1}}{p_{2} / p_{1}}
$$

where $\Theta_{i}$ is the loading, expressed say in molecules per unit cell, and $p_{i}$ is the partial pressure of the component $i$ in the bulk vapour phase. High sorption selectivities are desirable from the point of view of reducing equipment costs. Often in practice, the ratio of the Henry coefficients at zero loading is used to approximate the right-hand side of eqn. (1), i.e.

$$
S=\frac{H_{2}}{H_{1}}
$$

Consider a mixture of linear, mono-branched and dibranched alkanes in the 5-7 carbon atom range, of specific interest as ingredients of petrol, consisting of n-pentane $\left(\mathrm{n}-\mathrm{C}_{5}\right)$, n-hexane $\left(\mathrm{n}-\mathrm{C}_{6}\right)$, n-heptane (n-C $\left.\mathrm{C}_{7}\right), 2$-methylbutane $(2 \mathrm{MB}), 2$ methylpentane (2MP), 2-methylhexane (2MH), 2,2-dimethylbutane (22DMB), 2,2-dimethylpentane (22DMP). The sorption isotherms of these molecules in silicalite-1 calculated using configurational-bias Monte Carlo (CBMC) techniques, described in earlier work, ${ }^{4}$ for a temperature of $300 \mathrm{~K}$ are shown in Fig. 1. Firstly, let us consider the linear alkanes (Fig. 1(a)). Normal heptane shows a pronounced inflection at a loading of $\Theta=4$. Normal hexane shows a slight inflection at this loading due to "commensurate freezing" effects. ${ }^{4}$ All the three 2-methyl alkanes show inflection at a loading $\Theta=4$ (see Fig. 1(b)); this is because these molecules prefer to locate at the intersections between straight and zig-zag channels, which offers more "leg-room". ${ }^{3-5}$ At $\Theta=4$ all intersections are fully occupied. To locate the 2-methyl alkanes within the channel interiors requires an extra push, leading to inflection behaviour. The 22DMB and 22DMP molecules are too bulky to be located at the channel interiors and both molecules show a maximum (saturation) loading of 4 (see Fig. 1(c)). The Henry coefficients at $300 \mathrm{~K}$ in silicalite-1, calculated using the CBMC techniques (in the NVT framework), also described in earlier work, ${ }^{4}$ are shown in Fig. 2(a). On the basis of these Henry coefficients we would conclude that the hierarchy of sorption strengths roughly follows the hierarchy of $\mathrm{C}$ numbers; the higher the $\mathrm{C}$ number, the higher the adsorption strength. We would also conclude that it is not possible to separate the di-methyl alkanes selectively from the mixture.

In this paper we shall show that the use of Henry coefficients to determine the sorption hierarchy leads to completely erroneous results. With increasing loadings within the zeolite, 
(a) linear alkanes

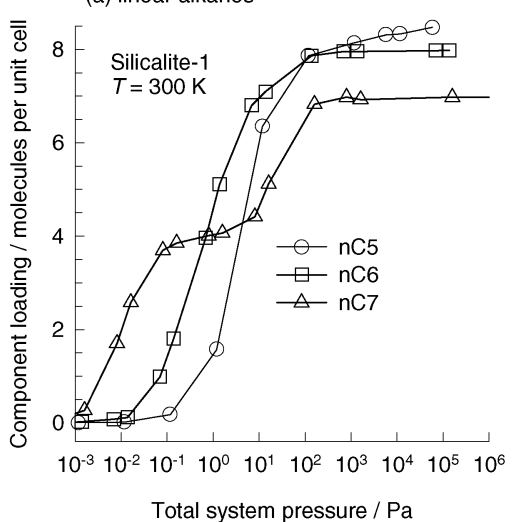

(b) 2-methylalkanes

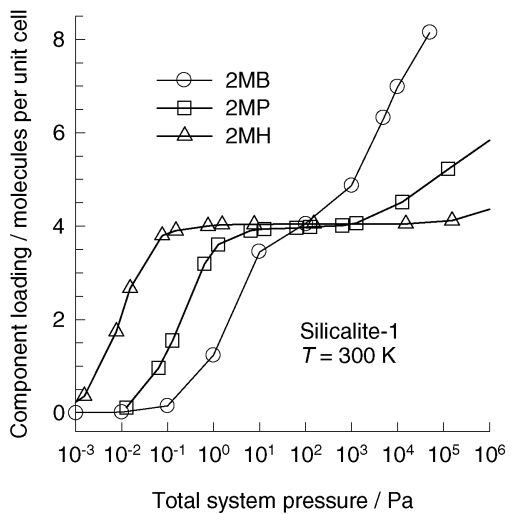

(c) double-branched alkanes

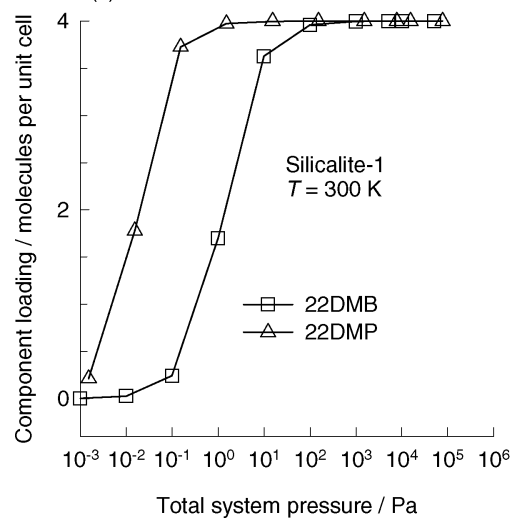

Fig. 1 Pure component isotherms for $\mathrm{n}-\mathrm{C}_{5}, \mathrm{n}-\mathrm{C}_{6}, \mathrm{n}-\mathrm{C}_{7}, 2 \mathrm{MB}, 2 \mathrm{MP}, 2 \mathrm{MH}, 22 \mathrm{DMB}$ and $22 \mathrm{DMP}$ at $300 \mathrm{~K}$ in silicalite calculated using CBMC simulations ${ }^{3-5}$

differences in molecular configuration play a significant role in determining sorption strengths. The "packing efficiency" of an alkane within the zeolite matrix decreases with increased degree of branching and follows the hierarchy linear alkanes $>$ mono-methyl alkanes $>$ di-methyl alkanes. That the linear molecules pack more efficiently within the silicalite structure is also evidenced by the differences in the saturation loadings, expressed in molecules per unit cell, between linear and branched alkanes in silicalite; see Fig. 2(b). Consider, for example, hexane isomers; the saturation loadings are as follows: $n-\mathrm{C}_{6}=8,2 \mathrm{MP}=6.2$ and $22 \mathrm{DMB}=4$ molecules per unit cell. We aim to demonstrate in this paper that for mixtures of linear and branched alkanes, in the 5-7 $\mathrm{C}$ atom range, such differences in packing efficiencies, i.e. configurational entropy effects, are strong enough to counteract, and overcome, chain-length effects. The net result is that the hierarchy of sorption strengths within the zeolite is linear alkanes $\gg$ mono-methyl alkanes $\gg$ di-methyl alkanes and could result in almost total exclusion of the di-branched isomer and extremely high sorption selectivities.

In our earlier publications ${ }^{3-5}$ we had used CBMC simulations to demonstrate that configurational entropy effects could be used to separate binary mixtures of linear and branched alkanes having the same number of $\mathrm{C}$ atoms. Here we extend the CBMC methodology to ternary and quaternary mixtures and study the interplay between chain length and configurational entropy effects. We show that entropy effects make it feasible to separate a mixture of $n-C_{5}-n-C_{6}-n-$ $\mathrm{C}_{7}-2 \mathrm{MB}-2 \mathrm{MP}-2 \mathrm{MH}-22 \mathrm{DMB}-22 \mathrm{DMP}$ into three fractions containing linear, mono-methyl and di-methyl alkanes, respectively.

For validation purposes, we note that though there is a considerable amount of published experimental data on pure component isotherms for various alkanes and isoalkanes, ${ }^{6-9}$ to our knowledge, there is only one study ${ }^{10}$ that presents ternary sorption data for linear, mono-methyl and di-methyl alkanes. The data of Santilli ${ }^{10}$ is used to validate the accuracy of our CBMC ternary simulations and establish the entropybased separation concept.

\section{Configurational-bias Monte Carlo (CBMC) simulation technique}

The linear and branched alkanes are described with a unitedatom model, in which $\mathrm{CH}_{3}, \mathrm{CH}_{2}$ and $\mathrm{CH}$ groups are considered as single interaction centers. When these pseudo-atoms belong to different molecules or to the same molecule but separated by more than three bonds, the interaction is given by a Lennard-Jones potential. The LennardJones parameters are chosen to reproduce the vapour-liquid curve of the phase diagram as shown in Siepmann et al. ${ }^{11}$ The bonded interactions include bond-bending and torsion potentials. Details for the alkane model can be found in Vlugt et $a .^{4}$

All the simulations have been performed in zeolite silicalite (MFI). Silicalite has both straight elliptical channels and zig-zag channels that cross at the intersections. Following Kiselev and co-workers, ${ }^{12}$ we consider the zeolite lattice to be (a) Henry coefficient

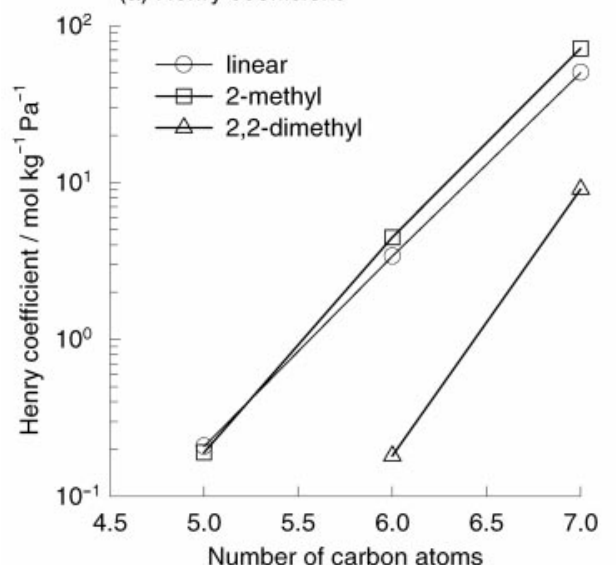

(b) maximum loading

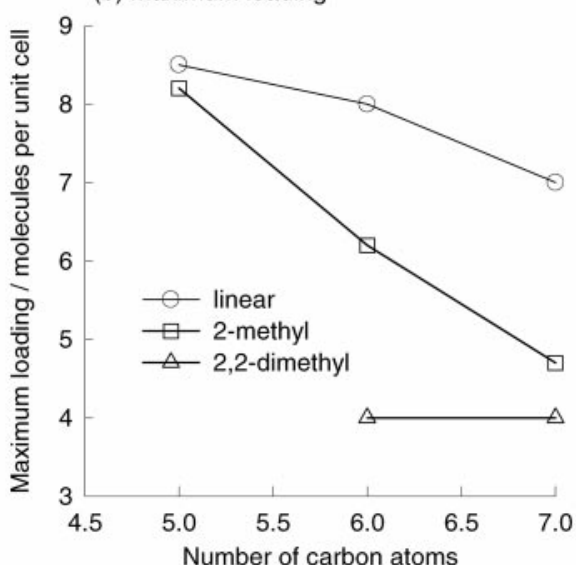

Fig. 2 (a) Henry coefficients of n-alkanes, 2-methyl alkanes and 2,2-dimethyl alkanes in silicalite. CBMC calculations at $300 \mathrm{~K}$ using NVT simulations. ${ }^{4}$ (b) Maximum saturation loadings of linear and branched alkanes. 
rigid and we assume that interactions of an alkane with the zeolite are dominated by the dispersive forces between alkane pseudo-atoms and the oxygen atoms of the zeolite. These interactions are described by a Lennard-Jones potential, the parameters of which are given in ref. 4.

Adsorption isotherms are conveniently computed using a Monte Carlo simulation in the grand-canonical ensemble. In this ensemble the temperature and chemical potentials are imposed. The average number of adsorbed molecules per unit cell of the zeolite follows from the simulations. The main characteristic of this type of simulations is that during the calculations attempts are made to change the total number of particles by making attempts to insert molecules into or remove molecules from the zeolite. To make such moves possible for the long chain alkanes we use the configurationalbias Monte Carlo (CBMC) technique. ${ }^{4,13}$ Instead of inserting a molecule at a random position, in a CBMC simulation a molecule is grown atom by atom in such a way that the "empty spots" in the zeolite are found. This growing scheme gives a bias that is removed exactly by adjusting the acceptance rules. ${ }^{4,13}$

These simulations were performed in cycles and in each cycle an attempt to perform one of the following moves was made:

(1) Displacement of a randomly selected chain. The maximum displacement was adjusted to an overall acceptance of $50 \%$.

(2) Rotation of a chain around its center-of-mass. The chain is chosen at random and the maximum rotation angle is selected to accept $50 \%$ of the moves.

(3) Partial regrowing of a chain; a chain is selected at random and part of the molecule is regrown using the CBMC scheme. It is decided at random which part of the chain is regrown and with which segment the regrowing is started. For branched alkanes, the approach in Vlugt et al. ${ }^{4}$ was used.

(4) Exchange with a reservoir; it is decided at random whether to add or to remove a molecule from the zeolite following the acceptance rules derived in Vlugt et al. ${ }^{4}$

(5) Change of identity (only in the case of mixtures); one of the components is selected at random and an attempt is made to change its identity. ${ }^{4}$ The acceptance rules for these trial moves can be found in Frenkel and Smit. ${ }^{13}$

The number of Monte Carlo cycles performed for one-, two-, three- and four-component isotherms were, respectively, $2 \times 10^{7}, 5 \times 10^{7}, 1 \times 10^{8}$ and $5 \times 10^{8}$. The relative probabilities of moves were: $15 \%$ displacements, $15 \%$ rotations, $15 \%$ partial re-growths, $50 \%$ exchanges with the reservoir and the remaining $5 \%$ of the moves were attempts to change the identity of a molecule. The number of trial orientations in the CBMC scheme was six for all molecules. In addition, we used the multiple first bead scheme with 15 trial positions for the first bead.

Two important aspects of our study, the assumption of a rigid silicalite lattice and the use of the united-atom model, deserve further discussion. Several authors have performed simulations on a flexible lattice (see the review by Demontis and Suffritti ${ }^{14}$ ). These simulations show that a flexible lattice can influence the diffusion properties. To diffuse through the zeolite the molecules have to pass through narrow windows that form energy barriers. In a flexible zeolite one may observe fluctuations in the size of the window that could lower this energy barrier to a significant extent. However, for the calculation of thermodynamic equilibrium properties, the focus of the present study, such fluctuations in the window size would have a much less significant effect because these involve much lower energy barriers. Therefore, we expect that, for simulation of adsorption isotherms, the use of a flexible zeolite would not change the results significantly. In earlier publications $^{4,5,15,16}$ we have made a detailed comparison of our CBMC model for adsorption of the pure components (linear and branched alkanes) in silicalite with experimental data. These studies showed that good agreement of CBMC simulations and experiments. We therefore conclude that it would be difficult to justify the use of flexible lattices in our simulations, especially when considering the additional computational expense.

A second aspect associated with the use of a flexible lattice is that the adsorbed molecule may induce a phase transition in the zeolite. Such a phase transition has been observed in H-ZSM5 for $p$-xylene, ${ }^{17}$ which molecules fit very snugly at the intersections of H-ZSM5. To the best of our knowledge such a transition has not been observed for the linear and branched alkanes; this can be understood from the fact that alkanes do not have such a tight fit in silicalite. Put another way, the sorption of linear and branched alkanes cannot be expected to induce phase transitions in the zeolite. A further implication is that the sorption properties of alkanes are not very sensitive to the choice of the effective channel, or window, size within the zeolite matrix. The effective channel or window size is determined by the positions of the atoms of the zeolite matrix, as determined from the crystal structure, and the zeolitealkane (Lennard-Jones) interactions. June et al. ${ }^{18}$ use a Lennard-Jones $\sigma$ value of $0.3364 \mathrm{~nm}$, whereas Vlugt et al. ${ }^{4}$ have chosen $0.360 \mathrm{~nm}$ in their CBMC simulations. Both set of parameters give reasonable results for sorption properties. The recent work of Vlugt et al. ${ }^{19}$ has, however, shown that the choice of $\sigma$ has a significant influence on the diffusion of isobutane in silicalite.

For the calculation of the sorption isotherms of alkanes in silicalite, Macedonia and Maginn ${ }^{20,21}$ have adopted an allatom model to represent the alkanes. Both the united-atom model (our approach) and the all-atom model give comparable results for the prediction of sorption of alkanes in silicalite. $^{20,21}$

\section{Separation of isomers of hexane}

Before considering chain length effects, let us first consider a mixture of hexane isomers: $n-C_{6}-3 M P-22 D M B$. Santilli ${ }^{10}$ has measured the component sorption loadings for an equimolar mixture at various temperatures in silicalite, keeping the total pressure constant. His data on the loadings of the individual molecules, and of the total mixture, have been replotted in Figs. 3(a) and 3(b). The behaviour of $n-C_{6}$ is "normal", inasmuch as increasing temperature, at constant total pressure, results in a decrease in the sorption loading. The behaviour of $3 \mathrm{MP}$ and 22DMB, in sharp contrast, are quite "abnormal"; these branched isomers initially show an increase in the loading with increasing temperature till a maximum is reached and beyond this point the loadings decrease with increasing temperature. In Fig. 3(c) the n$\mathrm{C}_{6} / 3 \mathrm{MP}$ and $\mathrm{n}-\mathrm{C}_{6} / 22 \mathrm{DMB}$ selectivities, defined as the ratio of the loadings, is plotted against temperature. We note that the selectivities approach unity at high temperatures and increase significantly as the temperature is reduced to below $400 \mathrm{~K}$. The increase in the selectivities is particularly steep when the total mixture loading, $\Theta_{\text {mix }}$, exceeds 4 molecules per unit cell.

The results presented in Fig. 3(c) have great practical potential because they show that it is possible to separate a mixture of hexane isomers, at high selectivities, provided the sorption conditions are maintained such that the mixture loading $\Theta_{\text {mix }}$ exceeds 4 molecules per unit cell. Let us rationalise the data of Santilli $^{10}$ by performing CBMC simulations. CBMC simulations for an equimolar mixture of $\mathrm{n}-\mathrm{C}_{6}, 3 \mathrm{MP}$ and $22 \mathrm{DMB}$ at $362 \mathrm{~K}$ are shown in Fig. 4(a) for a range of total pressures. The loadings of the three components follow "normal" behaviour for pressures below $50 \mathrm{~Pa}$ and the sorption loadings follow the hierarchy dictated by the Henry coefficients. At a pressure of $500 \mathrm{~Pa}, \Theta_{\text {mix }}=4$; at this loading all the intersection sites are fully occupied. At pressures exceeding $500 \mathrm{~Pa}$, 

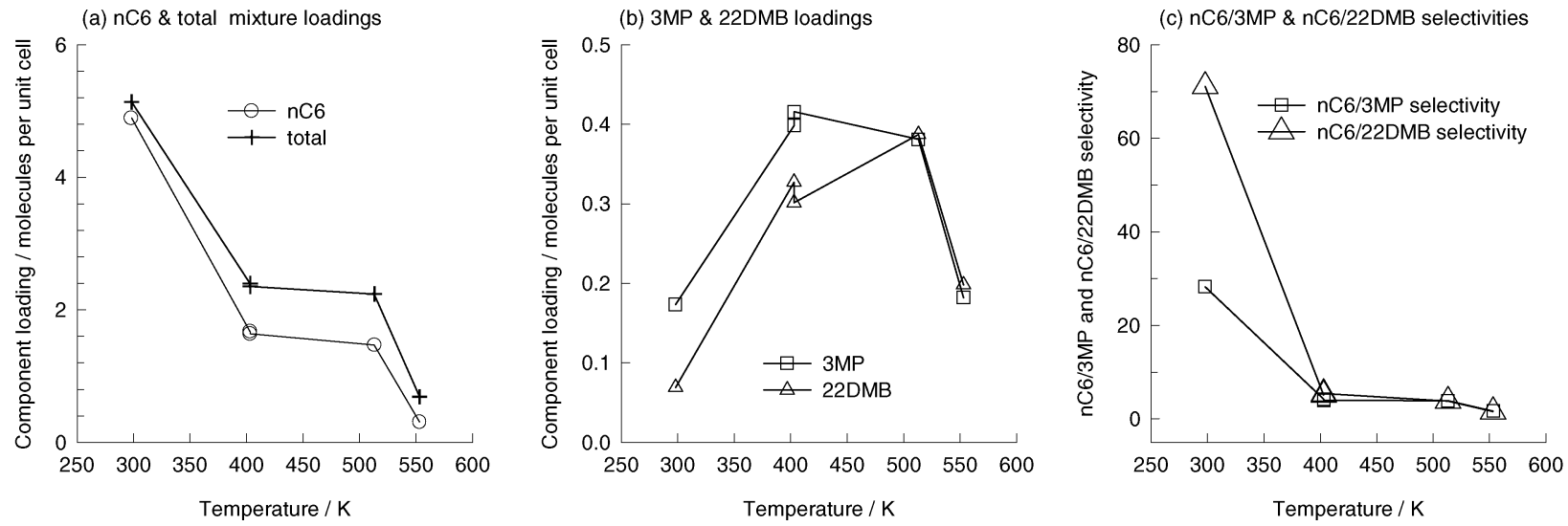

Fig. 3 (a) and (b) Component loadings for an equimolar mixture of $n-\mathrm{C}_{6}, 3 \mathrm{MP}$ and $22 \mathrm{DMB}$ as a function of temperature for constant pressure. (c) Sorption selectivity as a function of temperature. Experimental data of Santilli ${ }^{10}$ for silicalite.

the silicalite matrix prefers to accept $n-\mathrm{C}_{6}$ into its matrix rather than either of the branched isomers. The loadings of both $3 \mathrm{MP}$ and 22DMB decrease as the pressure is increased beyond $500 \mathrm{~Pa}$. There are three types of entropic "battles" in play at pressures exceeding $500 \mathrm{~Pa}$. The battle between linear isomer and its branched isomers is won by the linear isomer. The battle between the mono-methyl and di-methyl isomer is won by the mono-methyl isomer.

A more useful way to examine the ternary sorption data is to plot the component loadings against the total mixture loading $\Theta_{\text {mix }}$; see Fig. 4(b). We clearly note the maxima in the $3 \mathrm{MP}$ and 22DMB loadings when $\Theta_{\text {mix }}=4$. We also note that when $\Theta_{\text {mix }}>4$, the sorption loading hierarchy is $n-C_{6} \gg$ $3 \mathrm{MP} \gg 22 \mathrm{DMB}$. The $\mathrm{n}-\mathrm{C}_{6} / 3 \mathrm{MP}$ and $3 \mathrm{MP} / 22 \mathrm{DMB}$ sorption selectivities are shown in Fig. 4(c) as a function of $\Theta_{\text {mix }}$. We note the sharp increase in the sorption selectivities when $\Theta_{\text {mix }}>4$. For example, for $\Theta_{\text {mix }}=7$, both sorption selectivities are in excess of 100. Experimental evidence of the increase in sorption selectivity with increasing $\Theta_{\text {mix }}$ is contained in the data reported by Santilli ${ }^{10}$ and shown in Fig. 3(c) since a decreasing temperature, at constant pressure, corresponds to increasing $\Theta_{\text {mix }}$.

For direct comparison with the Santilli selectivity data, we performed a series of simulations at constant total pressure of $3 \mathrm{kPa}$ and temperatures of 300, 362, 400, 460, 510 and $550 \mathrm{~K}$. Fig. 5(a) compares the n-C6/3MP selectivity obtained from the CBMC simulations with those reported by Santilli. The two sets of selectivities are in excellent agreement. We may therefore conclude that the increase in selectivity with decreasing temperature is due to the fact that $\Theta_{\text {mix }}$ increases to values significantly higher than 4 when the temperature is decreased to below $400 \mathrm{~K}$. The Santilli data ${ }^{10}$ provide a direct confirmation of the concept of configurational entropy affecting mixture sorption and lends credence to our CBMC simulations.

In Fig. 5(b) the n-C6/22DMB sorption selectivity obtained from CBMC simulations is compared with the data of Santilli ${ }^{5}$ again reasonably good agreement is obtained. The strong increase in the selectivity when the temperature is reduced to below $400 \mathrm{~K}$ is again to be attributed to strong configurational entropy effects which favour the linear molecule to the di-branched molecules.

For direct comparison of the Santilli loading data with CBMC simulations, we plot the component loadings of the three hexane isomers as a function of $\Theta_{\text {mix }}$ in Figs. 6(a), 6(b) and $6(\mathrm{c})$. Shown in Fig. 6 are CBMC simulation results obtained at (i) $T=300,362,400,460,510,550 \mathrm{~K}$ and pressure $=3 \mathrm{kPa}$ and (ii) $T=362 \mathrm{~K}$ and a variety of pressures. Fig. 6(a) shows good agreement between the experimentally determined $n-C_{6}$ loadings and those calculated from CBMC. In Figs. 6(b) and 6(c) we note that the CBMC simulations predict a maxima in the 3MP and 22DMB loadings when $\Theta_{\text {mix }}=4$. Unfortunately the Santilli data is available only for $\Theta_{\text {mix }}=2$ and 6 molecules per unit cell. Nevertheless, the experiments confirm that there is a maximum in the loading of the branched alkane, $3 \mathrm{MP}$ and 22DMB, at $2<$ $\Theta_{\text {mix }}<6$ molecules per unit cell.

The results shown in Fig. 6 have practical implications. Provided the mixture loading $\Theta_{\text {mix }}$ is $>4$ molecules per unit cell, the sorption hierarchy is as follows: $\mathrm{n}-\mathrm{C}_{6} \gg 3 \mathrm{MP} \gg$ 22DMB, with the di-branched alkane being virtually excluded from the zeolite. The analysis presented above shows that it is
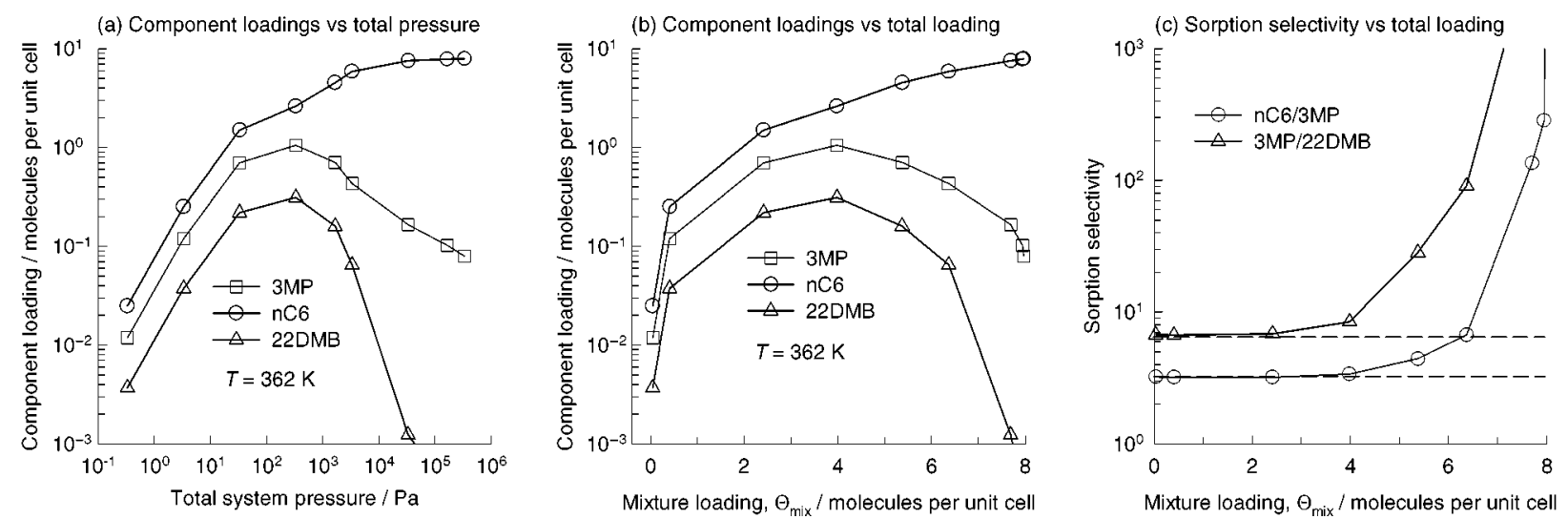

Fig. 4 (a) Component loadings for an equimolar mixture of $n-C_{6}-3 M P-22 D M B$ at $362 \mathrm{~K}$ determined by CBMC simulations for a variety of total system pressures. (b) Component loading vs. total mixture loading. (c) Sorption selectivities calculated from CBMC simulations are compared with the Henry selectivity, indicated by dashed lines. 

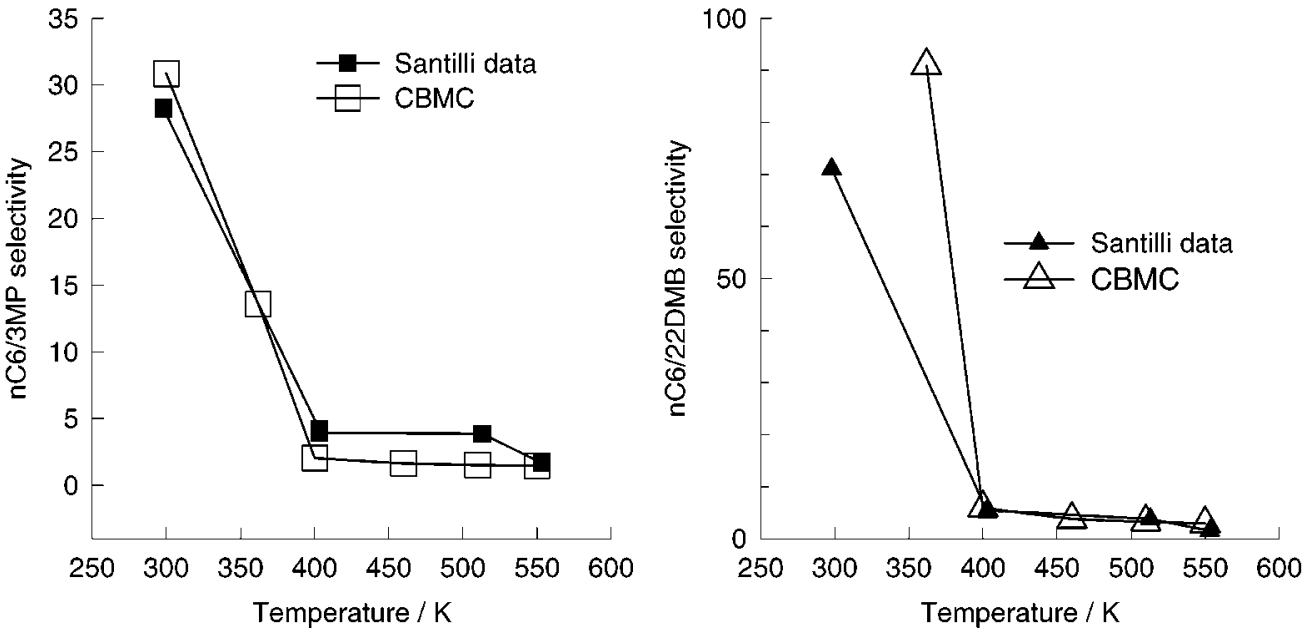

Fig. 5 Comparison of sorption sorption selectivity for (a) $n-C_{6} / 3 M P$ and (b) $n-C_{6} / 22 D M B$ in an equimolar ternary mixture of $n-$ $\mathrm{C}_{6}-3 \mathrm{MP}-22 \mathrm{DMB}$. The experimental data of Santilli1 ${ }^{10}$ are compared with calculations from CBMC simulations. The CBMC simulations were carried out at $T=300,362,400,460,510,550 \mathrm{~K}$ and total pressure $=3 \mathrm{kPa}$.

possible to separate hexane isomers with high selectivities into three fractions consisting of the linear, mono-methyl and dimethyl isomers.

\section{Separation of alkane mixtures in the 5-7 carbon atom range}

In industrial practice we have to contend with separations of mixtures of linear and branched alkanes in which the components differ in the number of $\mathrm{C}$ atoms. The mixture sorption characteristic is complicated because both chain length and configurational entropy effects play roles that counteract each other. Let us consider the specific problem of separating the mixture $n-\mathrm{C}_{5}-\mathrm{n}-\mathrm{C}_{6}-\mathrm{n}-\mathrm{C}_{7}-2 \mathrm{MB}-2 \mathrm{MP}-2 \mathrm{MH}-22 \mathrm{DMB}-$ 22DMP using silicalite, for which the pure component sorption isotherms at $300 \mathrm{~K}$ are shown in Fig. 1. We performed several CBMC simulations for various equimolar binary, ternary and quaternary mixtures to investigate the combination of chain length and configurational entropy effects.

\section{Sorption of equimolar binary mixtures}

Figs. 7(a), 7(b) and 7(c) show the CBMC simulations for n$\mathrm{C}_{5}-2 \mathrm{MB}, \mathrm{n}-\mathrm{C}_{6}-2 \mathrm{MP}$ and $\mathrm{n}-\mathrm{C}_{7}-2 \mathrm{MH}$ mixtures. For $\Theta_{\text {mix }}<4$ the isomers have practically the same sorption strength. However, for all the three isomeric mixtures, the loading of the mono-branched isomer reduces to very low values when $\Theta_{\text {mix }}>4$. The reason for this "exclusion" of the 2-methyl alkanes is because of configurational entropy effects which tend to favour the linear isomer.

For a binary mixture of mono-branched and di-branched alkanes, configurational entropy effects tend to favour the mono-branched alkane. This is evidenced in Fig. 7(d), which gives the sorption loadings for a binary mixture of $2 \mathrm{MH}$ and 22DMP. For $\Theta_{\text {mix }}>4$, the $2 \mathrm{MH}$ loading increases at the expense of 22DMP.

For a mixture of two linear alkanes the sorption loadings are primarily dictated by chain length considerations; the molecules with the longer chain length has a higher sorption strength; this is illustrated in Fig. 7(e) for $n-C_{5}-n-C_{6}$ mixture.

The sorption behaviour of a mixture of $n-C_{5}-2 M P$ is less intuitively obvious; the CBMC simulation results are shown in Fig. 7(f). At $\Theta_{\text {mix }} \ll 4,2 \mathrm{MP}$ sorption is favoured because of the chain length consideration. However, when $\Theta_{\text {mix }}=4$, all the intersection sites are fully occupied and the 2MP molecules demand an extra push to locate within the channel interiors. On the other hand, the $\mathrm{n}-\mathrm{C}_{5}$ molecules can be easily located everywhere within the silicalite matrix. For $\Theta_{\text {mix }}>4$, configurational entropy effects override the chain length considerations and the $n-C_{5}$ loading exceeds that of $2 \mathrm{MP}$. This selectivity reversal is important from a practical point of view and this effect would also be realised for $n-C_{6}-2 M H$ mixtures, for example.
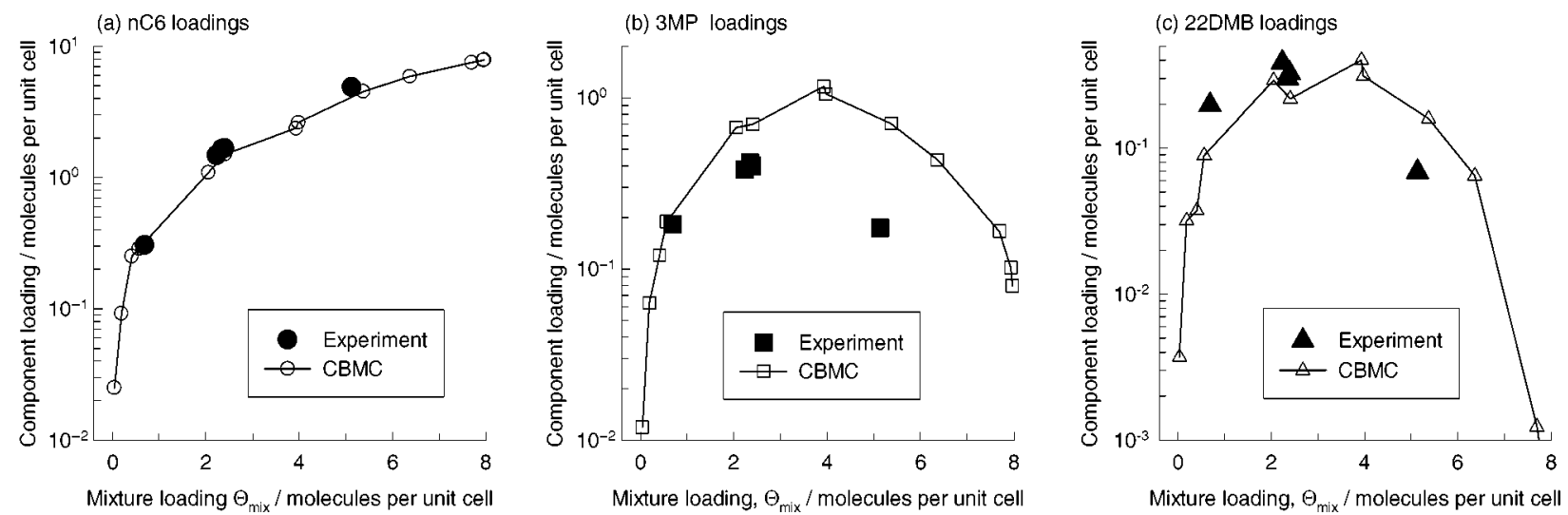

Fig. 6 Component loadings for an equimolar mixture of $n-C_{6}-3 M P-22 D M B$ as a function of total mixture loading. Experimental data of Santilli $^{10}$ are compared with CBMC simulations. The CBMC simulations were carried out at (i) $T=300,362,400,460,510,550 \mathrm{~K}$ and pressure $=3 \mathrm{kPa}$ and (ii) $T=362 \mathrm{~K}$ and a variety of pressures. 
(a) nC5 - 2MB equimolar mixture

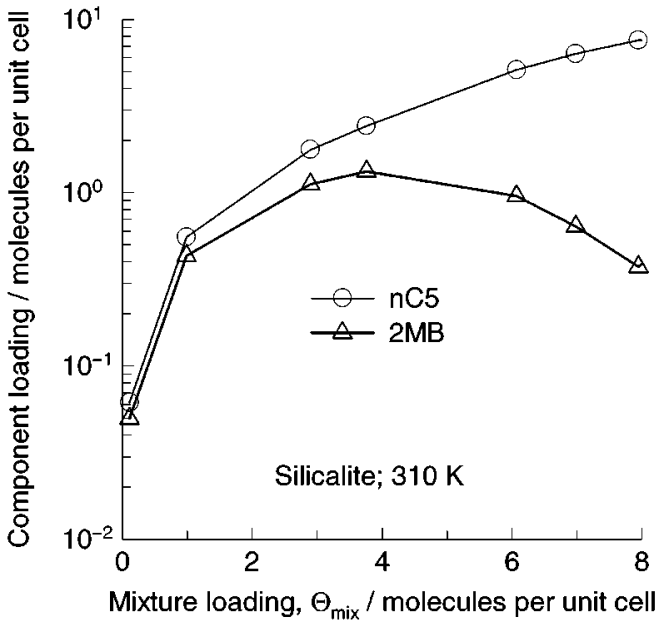

(c) $\mathrm{nC7}$ - $2 \mathrm{MH}$ equimolar mixture

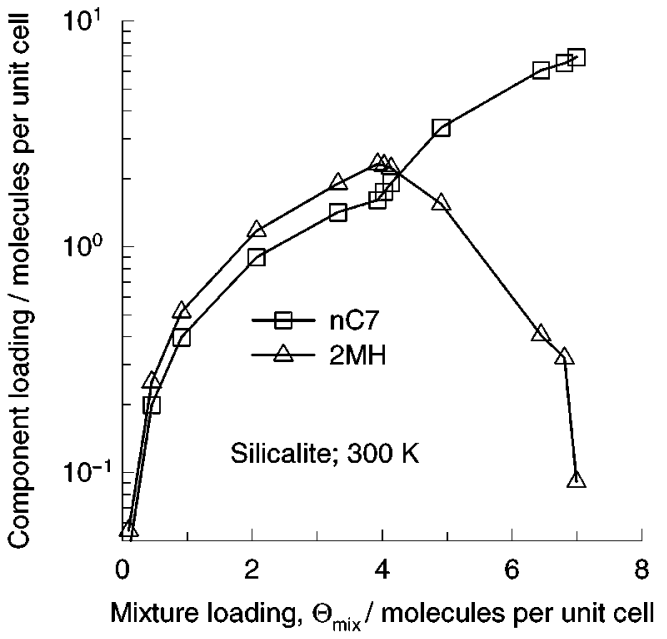

(e) nC5 - nC6 equimolar mixture

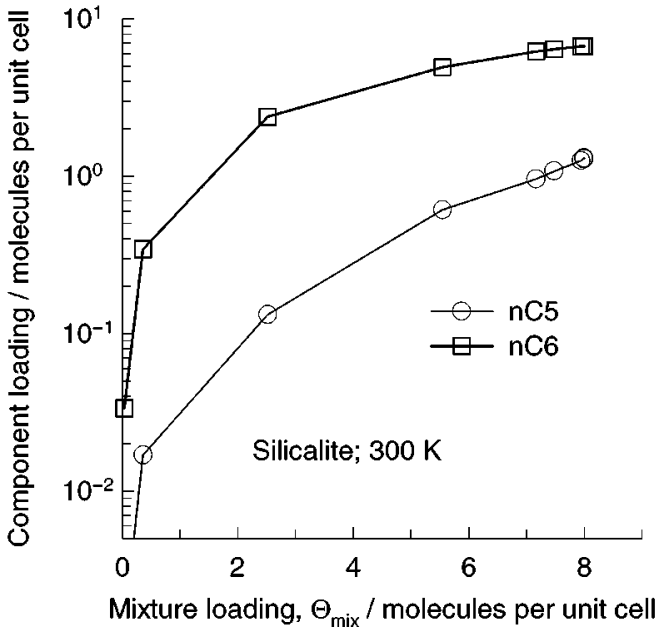

(b) nC6 - 2MP equimolar mixture

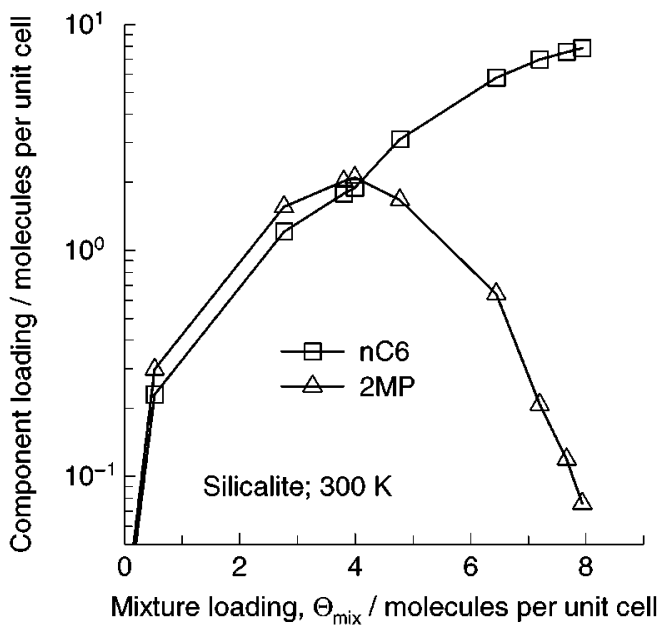

(d) $2 \mathrm{MH}-22 \mathrm{DMP}$ equimolar mixture

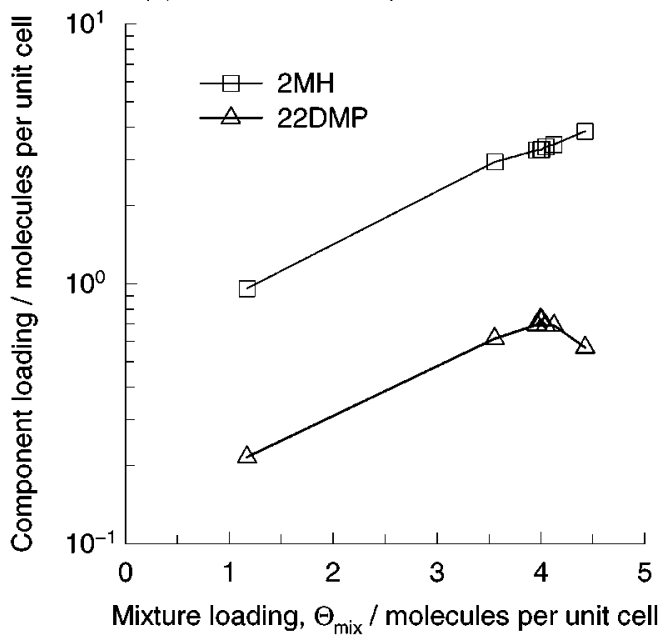

(f) nC5 - 2MP equimolar mixture

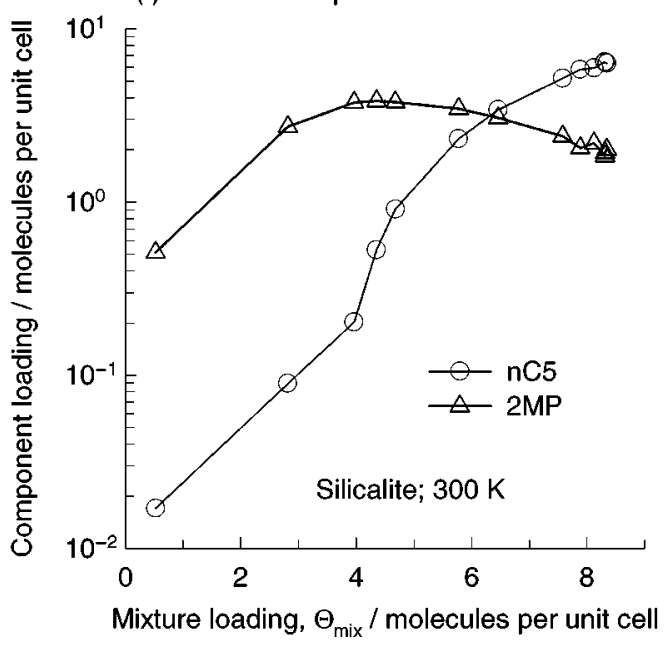

Fig. 7 Sorption loadings of various equimolar binary mixtures at $300 \mathrm{~K}$ in silicalite.

\section{Sorption of equimolar ternary mixtures}

CBMC simulations of the component loadings for an equimolar ternary mixture of $n-C_{5}-n-C_{6}-n-C_{7}$ at $300 \mathrm{~K}$ are shown in Fig. 8(a). For $\Theta_{\text {mix }}<4$, the sorption hierarchy is dictated by the $\mathrm{C}$ number, i.e. $\mathrm{n}-\mathrm{C}_{7}>\mathrm{n}-\mathrm{C}_{6}>\mathrm{n}-\mathrm{C}_{5}$. With increasing $\Theta_{\text {mix }}$ both chain length considerations and configurational entropy effects come into action. The $n-C_{7} / n-C_{6}$ selectivity decreases from 15 to values below unity because of configurational entropy effects which favour $\mathrm{n}-\mathrm{C}_{6}$; this is because $\mathrm{n}-\mathrm{C}_{7}$ exhibits inflection behaviour (see Fig. 1(a)). The $n-C_{6} / n-C_{5}$ selectivity decreases because of similar reasons. Near saturation loadings $\left(\Theta_{\text {mix }} \approx 8\right)$ the sorption hierarchy is $n-C_{6} \approx n$ $\mathrm{C}_{7}>\mathrm{n}-\mathrm{C}_{5}$.

Now consider a mixture of 2-methyl alkanes. CBMC simulations carried out for an equimolar mixture of $2 \mathrm{MB}, 2 \mathrm{MP}$ and $2 \mathrm{MH}$ at $300 \mathrm{~K}$ are shown in Fig. 8(b). All three molecules prefer to locate at the intersections. At $\Theta_{\text {mix }}=4$, all the inter- 
(a) nC5 - nC6 - nC7 equimolar mixture

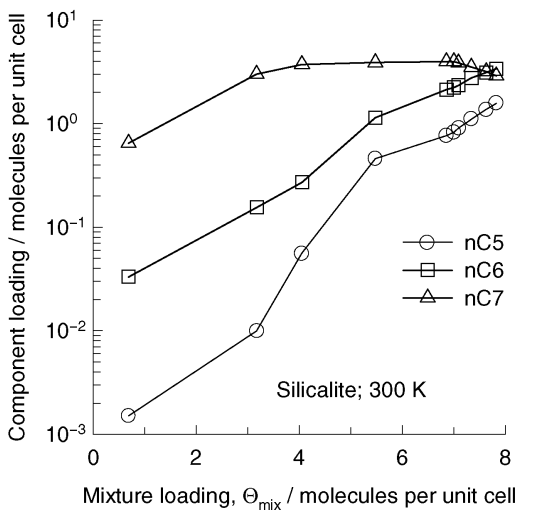

(b) $2 \mathrm{MB}$ - 2MP - $2 \mathrm{MH}$ equimolar mixture

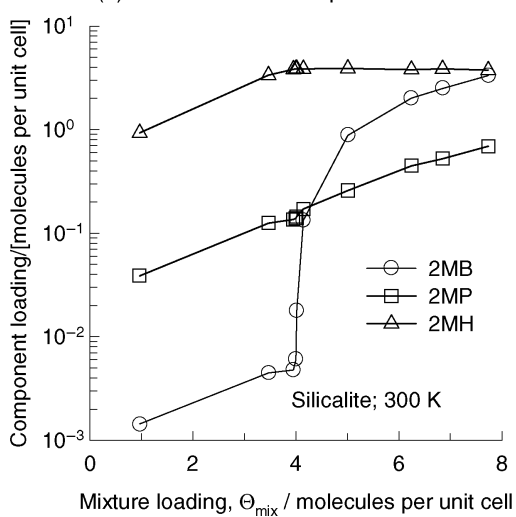

(c) nC5 - nC6 - 2MP equimolar mixture

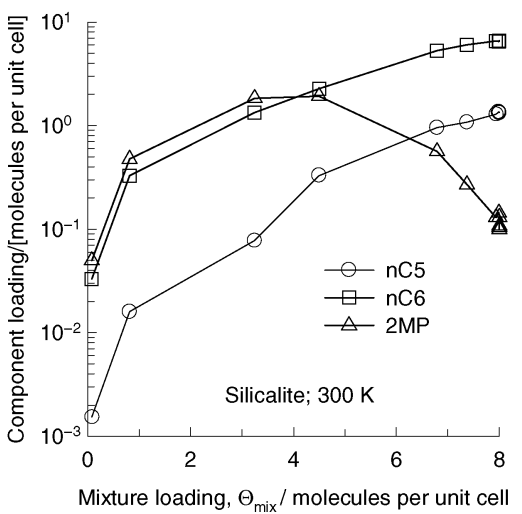

Fig. 8 Sorption loadings of various equimolar ternary mixtures at $300 \mathrm{~K}$ in silicalite.

sections are fully occupied. To locate the mono-branched molecules within the channel interiors an extra push is required; this push is highest for $2 \mathrm{MH}$, and decreases for $2 \mathrm{MP}$; see the pure component isotherms in Fig. 1(b). The $2 \mathrm{MB}$ molecules exhibit only a slight inflection (see Fig. 1(b)) and these molecules do not demand too much extra effort (i.e. pressure) to locate within the channel interiors. As a consequence the $2 \mathrm{MB}$ component loading increases sharply when the mixture loading exceeds 4 (cf. Fig. 8(b)). At saturation mixture loadings $\left(\Theta_{\text {mix }} \approx 8\right)$ it is interesting to note that the 2MB loadings exceeds that of $2 \mathrm{MP}$ and almost equals that of $2 \mathrm{MH}$. The CBMC simulation results in Fig. 8(b) underline the strong configurational entropy effects that tend to override chain considerations for a mixture of 2-methyl alkanes.

CBMC simulations for an equimolar mixture of $n-C_{5}, n-C_{6}$ and $2 \mathrm{MP}$ at $300 \mathrm{~K}$ are shown in Fig. 8(c). The loadings of the three components follow a "normal" behaviour for $\Theta_{\text {mix }}<2$ and the sorption loadings follow the hierarchy dictated by the Henry coefficients. When $\Theta_{\text {mix }}=4$ molecules per unit cell, all the intersection sites are occupied and from this point onward configurational entropy effects come into play. The 2MP loading shows a maximum at a mixture loading of 4 and decreases significantly when $\Theta_{\text {mix }}$ is increased. Configurational entropy effects serve to exclude the branched isomer from the silicalite matrix; it is more efficient to pack the zeolite with the two linear alkanes. When $\Theta_{\text {mix }}>6$, the $n-C_{5}$ loading exceeds that of $2 \mathrm{MP}$, demonstrating that configurational entropy effects dominate chain length considerations. The CBMC simulations show that, for this mixture, it is possible to separate both the linear alkanes from 2MP with high selectivity.

\section{Sorption of equimolar quaternary mixtures}

Consider an equimolar quaternary mixture of $n-C_{5}, n-C_{6}$, 2MB and 2MP. The CBMC simulations in Fig. 9(a) show that both mono-branched alkanes exhibit a maximum in their loadings when $\Theta_{\text {mix }}=4$. When $\Theta_{\text {mix }}$ is increased to near saturation (i.e. $\Theta_{\text {mix }} \approx 8$ ), the sorption hierarchy is $n-C_{6} \gg n-C_{5} \gg$ $2 \mathrm{MP} \gg 2 \mathrm{MB}$. An exactly analogous result is obtained for the quaternary mixture of $\mathrm{n}-\mathrm{C}_{5}, \mathrm{n}-\mathrm{C}_{7}, 2 \mathrm{MB}$ and $2 \mathrm{MH}$; see Fig. 9(b). In this case at near-saturation loadings the sorption hierarchy is $\mathrm{n}-\mathrm{C}_{7}>\mathrm{n}-\mathrm{C}_{5} \gg 2 \mathrm{MH} \gg 2 \mathrm{MB}$. It is remarkable to note that at saturation the sorption strength of $n-C_{5}$ exceeds that of $2 \mathrm{MH}$ even though its Henry coefficient is about 350 times smaller (cf. Fig. 2(a))! The results of Figs. 9(a) and 9(b) allow us to conclude that linear alkanes can be separated from mono-branched alkanes with high selectivities at nearsaturation loadings using silicalite.

Consider the quaternary mixture of mono- and di-branched alkanes: $2 \mathrm{MB}, 2 \mathrm{MP}, 2 \mathrm{MH}$ and 22DMP. CBMC simulations of the component loadings are shown in Fig. 9(c). The dibranched 22DMP suffers most from configurational entropy effects; note the maximum in the 22DMP loading when $\Theta_{\text {mix }}=4$. The smallest molecule, $2 \mathrm{MB}$, is the benefactor; its loading shows a sharp increase when the $\Theta_{\text {mix }}>4$ because all other molecules in the mixture need significant pushes to locate within the channel interiors. At saturation (i.e. $\Theta_{\text {mix }} \approx$ 8 ), the sorption hierarchy is $2 \mathrm{MH} \approx 2 \mathrm{MB} \gg 2 \mathrm{MP} \gg 22 \mathrm{DMP}$ and the di-branched 22DMP is practically excluded from the silicalite matrix. An exactly analogous result is obtained for the quaternary mixture of $2 \mathrm{MB}, 2 \mathrm{MP}, 2 \mathrm{MH}$ and $22 \mathrm{DMB}$; see Fig. $9($ d). In this case $22 \mathrm{DMB}$ is practically excluded from the silicalite matrix for saturation loadings. For the quaternary mixture of $2 \mathrm{MP}, 2 \mathrm{MH}, 22 \mathrm{DMB}$ and $22 \mathrm{DMP}$ both the dibranched alkanes exhibit maxima in the component loadings when $\Theta_{\text {mix }}=4$; see Fig. 9(e). At saturation, both the dibranched alkanes 22DMB and 22DMP are virtually excluded from the silicalite matrix. The CBMC simulation results shown in Figs. 9(c), 9(d) and 9(e) allow us to conclude that mono-branched alkanes can be separated from di-branched alkanes with high selectivities at near-saturation loadings (i.e. $\Theta_{\text {mix }} \approx 8$ ).

Finally, we consider the quaternary mixture of $n-C_{5}, 2 M B$, 2MP and 22DMP for which the CBMC simulation results for the component loadings are shown in Fig. 9(f). The dibranched 22DMP exhibits a maximum in the component loading when $\Theta_{\text {mix }}=4$; this molecule suffers the most from configurational entropy effects. The loadings of the monobranched $2 \mathrm{MP}$ and $2 \mathrm{MB}$ remain practically constant for $\Theta_{\text {mix }}>4$ and only the linear $\mathrm{n}-\mathrm{C}_{5}$ molecule increases in loading. At saturation (i.e. $\Theta_{\text {mix }} \approx 8$ ) the hierarchy of sorption loadings is $n-\mathrm{C}_{5} \gg 2 \mathrm{MP} \gg 2 \mathrm{MB} \gg 22 \mathrm{DMP}$, showing that it is possible to separate linear, mono-branched and di-branched alkanes with high selectivities.

\section{Concluding remarks}

We have examined the sorption characteristics of various mixtures of alkanes, in the 5-7 carbon atom range, in silicalite. The following major conclusions can be drawn:

1. For binary mixtures of linear and mono-branched alkanes with the same number of carbon atoms, the sorption selectivity increases in favour of the linear isomer for mixture loadings $\Theta_{\text {mix }}>4$; see Figs. 7(a), 7(b) and 7(c). This is due to configurational entropy effects. This effect is so strong that the mono-branched alkanes are virtually excluded from the silicalite matrix at saturation loadings.

2. For binary mixtures of mono-branched and di-branched alkanes with the same number of carbon atoms, the sorption selectivity increases in favour of the mono-branched isomer for $\Theta_{\text {mix }}>4$; see Fig. 7(d). This is also due to configurational entropy effects. 
(a) nC5 - nC6 - 2MB - 2MP equimolar mixture

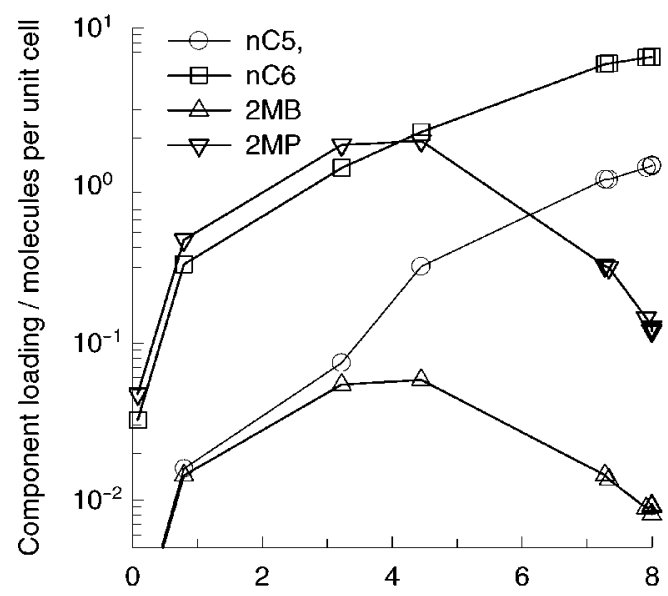

Mixture loading, $\Theta_{\text {mix }} /$ molecules per unit cell

(c) 2MB - 2MP - 2MH - 22DMP equimolar mixture

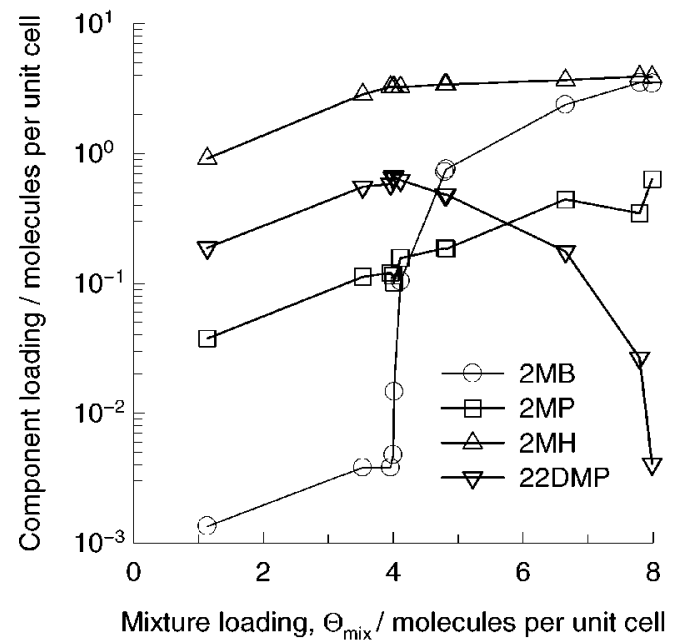

(e) 2MP - 22DMB - 2MH - 22DMP equimolar mixture

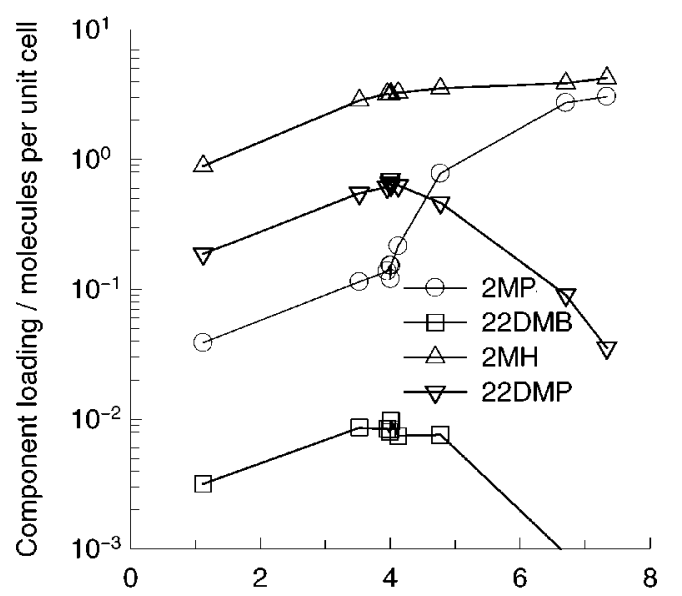

Mixture loading, $\Theta_{\text {mix }} /$ molecules per unit cell (b) $\mathrm{nC} 5$ - 2MB - $\mathrm{nC} 7-2 \mathrm{MH}$ equimolar mixture

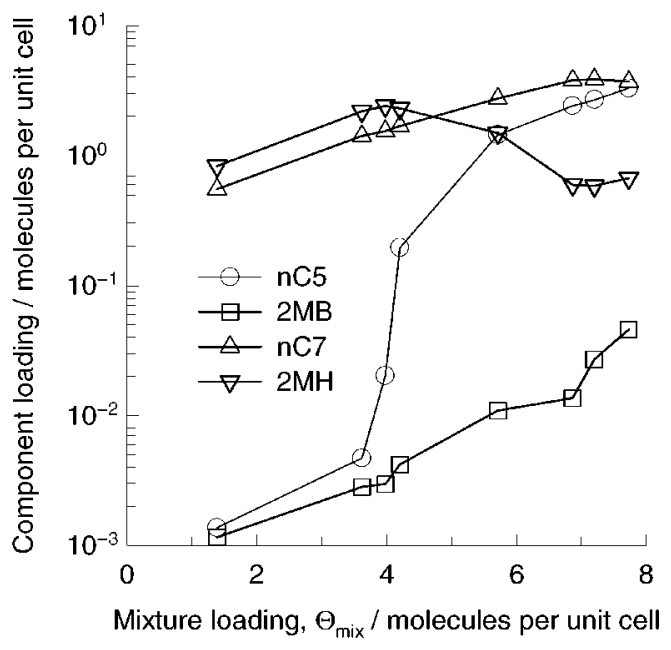

(d) 2MB - 2MP - 22DMB -2MH equimolar mixture

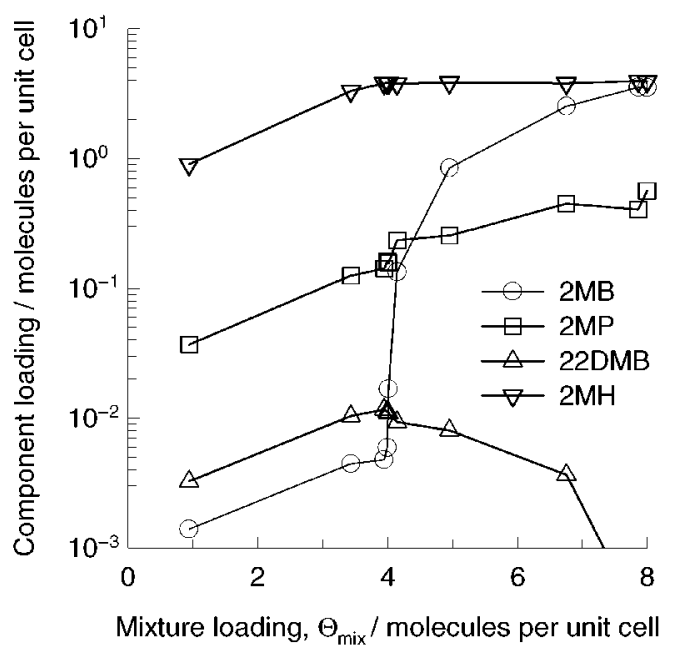

(f) $\mathrm{nC5}$ - 2MB - 2MP - 22DMB equimolar mixture

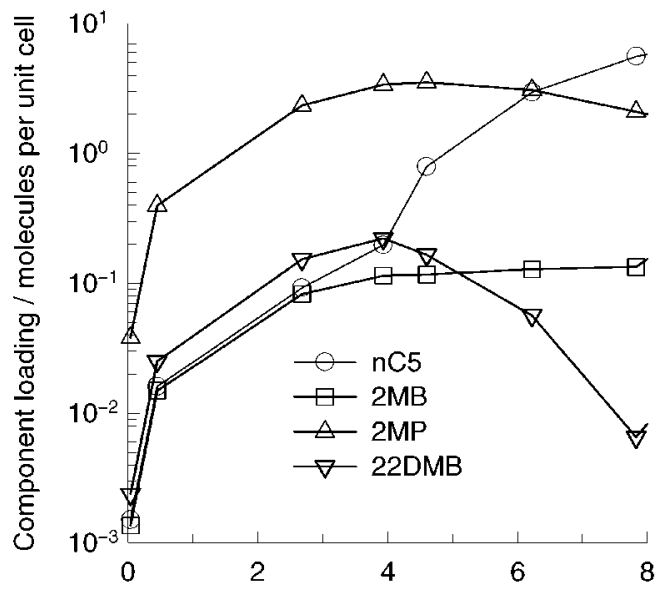

Mixture loading, $\Theta_{\text {mix }} /$ molecules per unit cell

Fig. 9 Sorption loadings of various equimolar quaternary mixtures at $300 \mathrm{~K}$ in silicalite.

3. For a mixture of linear alkanes the sorption hierarchy normally favours the molecule with the longer chain length; see Figs. 7(e) and 8(a). However, at saturation conditions $\mathrm{n}^{-\mathrm{C}_{7}}$ suffers a configurational entropy penalty and its sorption strength is lower than that of $n-C_{6}$; see Fig. 8(a).

4. For a ternary mixture of mono-branched alkanes, $2 \mathrm{MB}$,
$2 \mathrm{MP}$ and $2 \mathrm{MH}$, configurational entropy affects the sorption loading of all three molecules when $\Theta_{\text {mix }}>4$. 2MH suffers the most and $2 \mathrm{MB}$ is the benefactor; see Fig. $8(\mathrm{~b})$.

5. For mixtures of linear, mono-branched and di-branched alkanes with the same number of carbon atoms, the sorption selectivity increases in favour of the linear isomer for $\Theta_{\text {mix }}>4$. 
This is due to configurational entropy effects. This effect is so strong that the mono- and di-branched alkanes are virtually excluded from the silicalite matrix and high separation factors are achievable. This is confirmed by the experimental data of Santilli $^{10}$ for the ternary mixture $n-C_{6}-3 M P-22 D M B$; see Figs. 3-6.

6. From the quaternary simulation results presented in Figs. 9(a) and 9(b) we conclude that it is possible to separate linear alkanes from mono-branched alkanes with high selectivities provided we operate near saturation loading conditions (i.e. $\Theta_{\text {mix }} \approx 8$ ).

7. From the quaternary simulation results presented in Figs. 9(c), 9(d) and 9(e) we conclude that it is possible to separate mono-branched alkanes from di-branched alkanes with high selectivities provided we operate near saturation loading conditions (i.e. $\Theta_{\text {mix }} \approx 8$ ).

8. From the quaternary simulation results presented in Fig. 9(f) we conclude that it is possible to separate linear, monobranched alkanes and di-branched alkanes from one another with high selectivities provided we operate near saturation loading conditions (i.e. $\Theta_{\text {mix }} \approx 8$ ).

Our CBMC simulation results allow the development of a novel process for the separation of linear, mono-branched and di-branched alkanes in the 5-7 $\mathrm{C}$ atom range using silicalite. The main clue is to operate at high mixture loadings, approaching saturation (i.e. $\Theta_{\text {mix }} \approx 8$ ), and exploitation of configurational entropy effects. There is some evidence in the patent literature, ${ }^{1}$ that such entropy effects are being harnessed in industrial practice.

\section{Acknowledgements}

All the authors of this study have received grants from the Netherlands Organisation for Scientific Research (NWO), through the Netherlands Research Council for Chemical Sciences $(\mathrm{CW})$.

\section{References}

1 H. W. Dandekar, G. A. Funk and H. A. Zinnen, US Pat. 6069289 to UOP, Inc., 2000.

2 J. F. Denayer, G. V. Baron, J. A. Martens and P. A. Jacobs, J. Phys. Chem. B, 1998, 102, 3077.

3 R. Krishna, B. Smit and T. J. H. Vlugt, J. Phys. Chem. A, 1998, $102,7727$.

4 T. J. H. Vlugt, R. Krishna and B. Smit, J. Phys. Chem. B, 1999, 103, 1102.

5 M. Schenk, S. L. Vidal, T. J. H. Vlugt, B. Smit and R. Krishna, Langmuir, 2001, 17, 1558.

6 L. Boulicaut, S. Brandani and D. M. Ruthven, Microporous Mesoporous Mater., 1998, 25, 81.

7 C. L. Cavalcante and D. M. Ruthven, Ind. Eng. Chem. Res., 1995, 35, 177.

8 M. S. Sun, O. Talu and D. B. Shah, J. Phys. Chem., 1996, 100, 17276.

9 W. Zhu, F. Kapteijn and J.A. Moulijn, Phys. Chem. Chem. Phys., 2000, 2, 1989

10 D. S. Santilli, J. Catal., 1986, 99, 335.

11 J. I. Siepmann, M. G. Martin, C. J. Mundy and M. L. Klein, Mol. Phys., 1997, 90, 687

12 A. G. Bezus, A. V. Kiselev, A. A. Lopatkin and P. Q. Du, J. Chem. Soc., Faraday Trans. 2, 1978, 74, 367.

13 D. Frenkel and B. Smit, Understanding Molecular Simulations: from Algorithms to Applications, Academic Press, San Diego, 1996.

14 P. Demontis and G. B. Suffritti, Chem. Rev., 1997, 97, 2845.

15 Z. Du, G. Manos, T. J. H. Vlugt and B. Smit, A.I.Ch.E.J., 1998, 44, 1756.

16 R. Krishna and D. Paschek, Phys. Chem. Chem. Phys., 2001, 3, 453.

17 H. Van Koningsveld, F. Tuinstra, H. Van Bekkum and J. C. Jansen, Acta Crystallogr. Sect. B, 1989, 45, 423.

18 R. L. June, A. T. Bell and D. N. Theodorou, J. Phys. Chem., 1992, 96, 1051.

19 T. J. H. Vlugt, C. Dellago and B. Smit, J. Chem. Phys., 2000, 113, 8791.

20 M. D. Macedonia and E. J. Maginn, Fluid Phase Equilib., 1999, 160, 19.

21 M. D. Macedonia and E. J. Maginn, Mol. Phys., 1999, 96, 1375. 